Research Article

\title{
Tetrahydroxystilbene Glucoside Produces Neuroprotection against 6-OHDA-Induced Dopamine Neurotoxicity
}

\author{
Chun Huang, Fengqin Lin, Guoqing Wang, Disheng Lu, Qin Wu, Jie Liu $\mathbb{D}$, Jingshan Shi, \\ and Feng Zhang $\mathbb{B}$
}

Joint International Research Laboratory of Ethnomedicine of Ministry of Education and Key Laboratory of Basic Pharmacology of Ministry of Education, Zunyi Medical University, Zunyi, Guizhou, China

Correspondence should be addressed to Feng Zhang; zhangfengzmc@163.com

Received 16 March 2017; Revised 7 July 2017; Accepted 11 December 2017; Published 14 January 2018

Academic Editor: Fakhrul Islam

Copyright (C) 2018 Chun Huang et al. This is an open access article distributed under the Creative Commons Attribution License, which permits unrestricted use, distribution, and reproduction in any medium, provided the original work is properly cited.

Parkinson's disease (PD) was one of the most common neurodegenerative diseases with a slow and progressive loss of dopamine (DA) neurons in the midbrain substantia nigra (SN). Neuroinflammation was identified to be an important contributor to PD pathogenesis with the hallmark of microglia activation. Tetrahydroxystilbene glucoside (TSG) was the main active component extracted from Polygonum multiflorum and held amounts of pharmacological activities including antioxidant, free radicalscavenging, anti-inflammation, and cardioprotective properties. Recent studies demonstrated that TSG exerted neuroprotection from several neurodegenerative disease models. However, the underlying mechanisms were not completely elucidated. In the present study, rat nigral stereotaxic injection of 6-hydroxydopamine- (6-OHDA-) elicited DA neuronal injury was performed to investigate TSG-mediated neuroprotection on DA neurons. In addition, primary rat midbrain neuron-glia cocultures were applied to explore the mechanisms underlying TSG-exerted neuroprotection. Results showed that daily intraperitoneal injection of TSG for 14 consecutive days significantly protected DA neurons from 6-OHDA-induced neurotoxicity and suppressed microglia activation. Similar neuroprotection was shown in primary neuron-glia cocultures. In vitro studies further demonstrated that TSG inhibited microglia activation and subsequent release of proinflammatory factors. Moreover, TSGmediated neuroprotection was closely related with the inactivation of mitogen-activated protein kinase (MAPK) signaling pathway. Together, TSG protects DA neurons from 6-OHDA-induced neurotoxicity via the inhibition of microglia-elicited neuroinflammation. These findings suggest that TSG might hold potential therapeutic effects on PD.

\section{Introduction}

Parkinson's disease (PD) is the second most prevalent neurodegenerative disease characterized by a selective loss of dopamine (DA) neurons in the midbrain substantia nigra (SN) $[1,2]$. Although the pathogenesis of PD is not completely elucidated, microglia-mediated neuroinflammation has attracted increasing attention [3]. Microglia are the resident immune cells in the brain, acting as the first line of defense from external stimuli. Once activated by brain damage or immune stimuli, microglia release amounts of proinflammatory factors, such as interleukin $1 \beta$ (IL-1 $\beta)$, nitric oxide (NO), and tumor necrosis factor $\alpha$ (TNF- $\alpha$ ). The accumulation of these factors led to the surrounded DA neuronal injury [4]. Taken together, microglia-induced neuroinflammation might be a key event in the degradation of DA neurons and the inhibition of microglia activation could possess a promising therapeutic potential for PD [5].

Tetrahydroxystilbene glucoside (TSG), the main active component derived from Polygonum multiflorum, holds a large amount of pharmacological properties including antioxidant, free radical-scavenging, anti-inflammation, and cardioprotective effects [6]. Current evidence indicated that TSG has significant neuroprotective properties on ischemic brain both in vitro and in vivo [7]. Additionally, TSG could not only improve memory and motor function but also 
attenuate $\alpha$-synuclein aggregation in the striatum of aged mice $[8,9]$. However, the mechanisms through which TSGexerted neuroprotection are not completely elucidated. In the present study, rat nigral stereotaxic injection of 6-hydroxydopamine- (6-OHDA-) elicited DA neuronal injury was performed to investigate TSG-mediated neuroprotection on DA neurons. Furthermore, primary rat midbrain neuronglia cocultures were applied to explore the mechanisms underlying TSG-produced neuroprotection.

\section{Materials and Methods}

2.1. Reagents. TSG (purity $>99 \%$ ) was obtained from the National Institute for the Control of Pharmaceutical and Biological Products (Beijing, China). 6-OHDA was bought from Sigma-Aldrich (St. Louis, MO, USA). Anti-CR3 complement receptor (OX-42) and tyrosine hydroxylase (TH) antibodies were bought from Abcam Inc. (Cambridge, MA, USA). Biotinylated secondary antibodies were from Vector Laboratories (Burlingame, CA, USA). Enzymelinked immunosorbent assay (ELISA) kits were from R\&D Systems (Minneapolis, MN, USA). Griess reagent was obtained from Beyotime Biotechnology (Shanghai, China). Mitogen-activated protein kinase (MAPK) pathway antibodies were the products of Cell Signaling Technology (Beverly, MA, USA).

2.2. Animals and Treatment. Male Wistar rats (200-250 g) were bought from the Experimental Animal Center in the Third Military Medical University. Animal breeding and housing were strictly performed in the accordance with the Animal Care and Use Guidelines of China. To investigate the neuroprotective actions of TSG on 6-OHDA-elicited neurotoxicity, male rats were randomly divided into control, TSG (50 mg/kg) alone, 6-OHDA group, 6-OHDA + TSG $(10 \mathrm{mg} / \mathrm{kg})$, and $6-\mathrm{OHDA}+\mathrm{TSG}(50 \mathrm{mg} / \mathrm{kg})$ groups. Rats received a single 6-OHDA injection $(8 \mu \mathrm{g})$ into the midbrain $\mathrm{SN}$ on the left side of the brain, followed by the coordinates $5.2 \mathrm{~mm}$ posterior to bregma, $2.4 \mathrm{~mm}$ lateral to the midline, and $8.0 \mathrm{~mm}$ ventral to the surface of the skull [10]. Then, TSG was intraperitoneally injected into rats daily for 14 consecutive days. After the last TSG treatment, rats were allowed to recover for an additional 2 weeks development and then the behavior tests (rotarod test) were applied. Afterwards, animals were sacrificed and the biochemical analysis was performed.

2.3. Rotarod Test. Rotarod test was performed to study the muscular coordination. It contained cylindrical arrangement of the thin steel rods with two parts by compartmentalization to permit the detection of two rats at the same time. In the train, the speed was set at $10 \mathrm{cycles} / \mathrm{min}$ and the cutoff time was $180 \mathrm{~s}$. Before the start of the test, rats were trained on the rotarod until they remained on the rod no less than the cutoff time [11]. Animals were allowed to keep stationary for a while at $0 \mathrm{rpm}$. The rotational speed was gradually increased to $10 \mathrm{rpm}$ in $20 \mathrm{~s}$ interval till rats fell off the rungs. Animals were detected for two trials per day, and the mean duration time that they remained on the rod was recorded.
2.4. High-Performance Liquid Chromatography (HPLC) Coupled with Electrochemical Detection. Rat striatum levels of DA and its metabolite, 3,4-dihydroxyphenylacetic acid (DOPAC), were measured by HPLC coupled with electrochemical detection. Rat striatum tissues were sonicated in the perchloric acid including the internal standard 3,4-dihydroxybenzylamine. The homogenate was centrifuged, and an aliquot of the supernatant was injected into the HPLC. The mobile phase consisted of acetonitrile, tetrahydrofuran, and monochloroacetic acid ( $\mathrm{pH} 3.0)$ containing ethylenediaminetetraacetic acid sodium octyl sulfate $(200 \mathrm{mg} / \mathrm{l})$ and (EDTA, $50 \mathrm{mg} / \mathrm{l})$. The DA and DOPAC levels were detected and expressed in the quality of wet weight of tissue [12].

2.5. Immunohistochemical Analysis and Cell Counting in the $S N$. Rat brains were cut with a horizontal sliding microtome into $35 \mu \mathrm{m}$ transverse free-floating sections. A total of 36 consecutive brain slices throughout the entire SN were collected, in which every 6 th section was performed for the immunocytochemical analysis. DA neuron was recognized by an anti-tyrosine hydroxylase (TH) antibody, and the activated microglia were identified with an antiOX-42 antibody. Digital images of TH-positive neurons and OX-42-positive microglia in midbrain SN were acquired by an Olympus microscope (Olympus, Tokyo, Japan). The DA neurotoxicity was evaluated by $\mathrm{TH}$-positive neuron counting, and microglia activation-elicited neuroinflammation was detected by densitometry assessment of OX-42positive microglia.

2.6. Primary Rat Midbrain Neuron-Glia Cocultures. Primary rat neuron-glia cocultures were prepared from the midbrain tissues of embryonic day 14 and 15 rats [12]. In brief, the whole brain was aseptically removed and the mesencephalon was dissected. After removing the blood vessels and meninges, the midbrain tissues were dissociated with a mild mechanical trituration. Then, the dissociated cells were seeded at $5 \times 10^{5} /$ well in a 24 -well plate. The cocultures were maintained in a humidified atmosphere of $5 \% \mathrm{CO}_{2}$ and $95 \%$ air at $37^{\circ} \mathrm{C}$ with the maintenance medium. The seven-day-old cocultures were applied for drug treatments. At the time of treatment, cultures were composed of $50 \%$ astroglia, $10 \%$ microglia, and $40 \%$ neurons (including $1 \%$ DA neurons) [13].

2.7. Immunocytochemical Staining. Formaldehyde (4\%)-fixed cells were treated with hydrogen peroxide (1\%) and then incubated with the blocking solution. Cultures were incubated at $4^{\circ} \mathrm{C}$ overnight with primary anti-OX-42 (1:300) and anti-TH $(1: 300)$ antibodies followed by the biotinylated secondary antibody and Vectastain $\mathrm{ABC}$ reagents. Color was developed with 3,3'-diaminobenzidine. For the visual THpositive neuron counting, four representative areas per well of the 24-well plate were counted. In each condition, three wells were applied for cell counting.

2.8. IL-1 $\beta$, TNF- $\alpha$, and Nitrite Assay. The TNF- $\alpha$ and IL- $1 \beta$ levels in the supernatant were determined by ELISA kits. The release of NO was analyzed by detecting the accumulated 

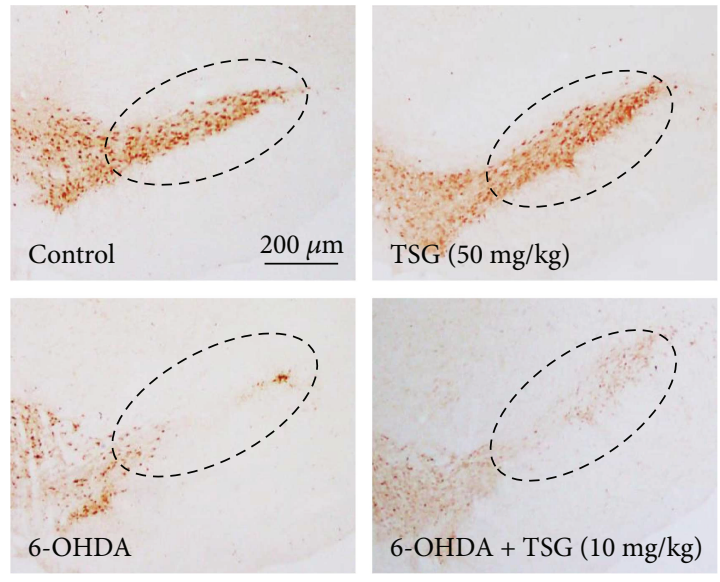

(a)
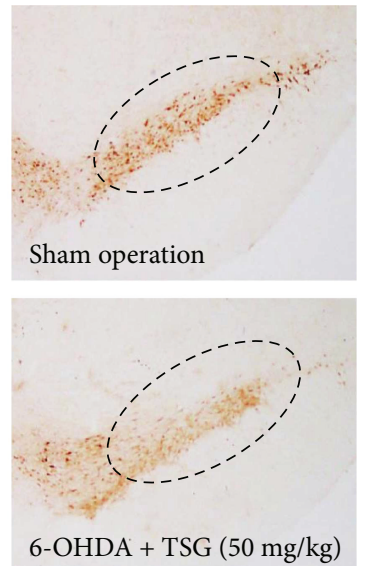

6-OHDA + TSG $(50 \mathrm{mg} / \mathrm{kg})$

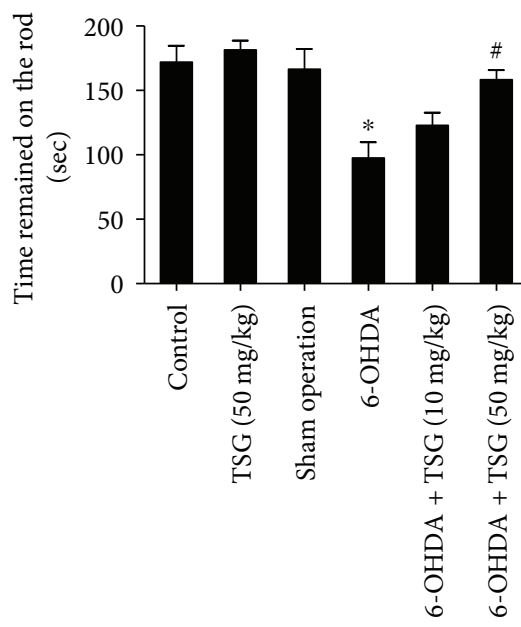

(c)

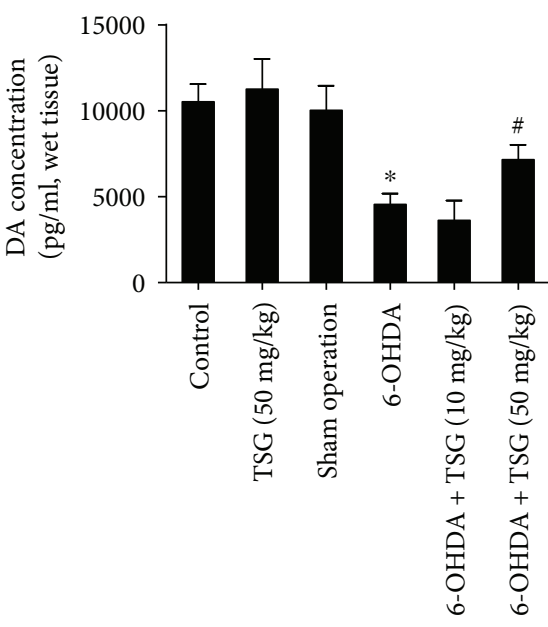

(d)

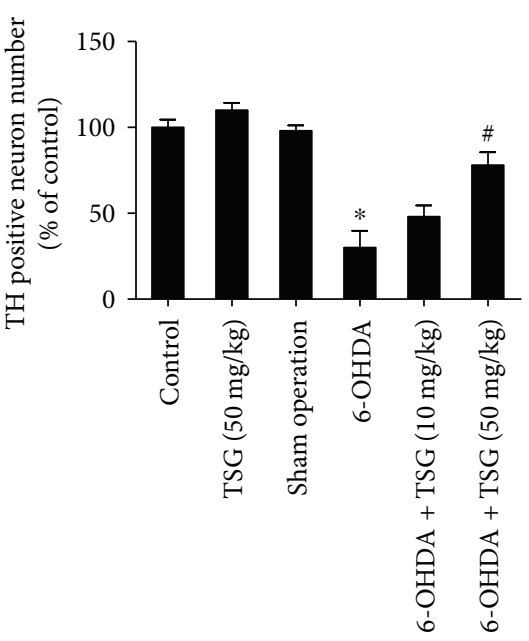

(b)

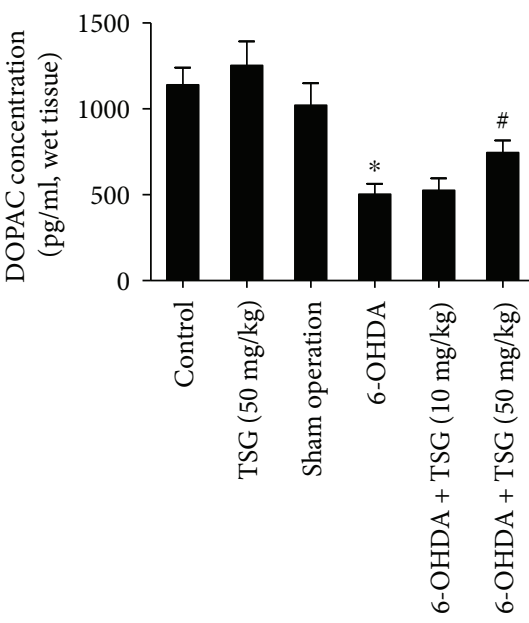

(e)

FIgure 1: TSG inhibited 6-OHDA-induced DA neuronal loss in the SN in vivo. Brain sections were immunostained with an anti-TH antibody (a). The "ellipse" presented the area of SN. Scale bar $=200 \mu \mathrm{m}$. The number of TH-positive neurons in the SN was counted (b). Rat behavior changes were analyzed by rotarod test. The time that the rats remained on the rod was recorded (c). The DA (d) and DOPAC (e) levels in rat brain striatum were measured by HPLC coupled with electrochemical detection. Results were expressed as a percentage of the control group and were the mean \pm SEM from six rats. ${ }^{*} p<0.05$ compared with the control groups; ${ }^{\#} p<0.05$ compared with 6-OHDA-treated group.

levels of nitrite (an indicator of NO) with Griess reagent in the medium of neuron-glia cocultures.

2.9. Western Blot Analysis. For the whole cell lysate extraction, cultures were homogenized in RIPA lysis buffer. The lysates were incubated on ice for $30 \mathrm{~min}$ and then centrifuged at $12000 \times \mathrm{g}$ for $15 \mathrm{~min}$. Protein concentrations were measured using BCA assay. Equal amounts of total protein were separated on 4-12\% Bis-Tris Nu-PAGE gel and then transferred to PVDF membranes. Subsequently, the membranes were blocked by $5 \%$ nonfat milk and then incubated with the following primary antibodies: ionized calciumbinding adapter molecule-1 (Iba-1, 1:800), TH (1:500), phosphorylated-p38 (p-p38, 1:1000), p38 (1:1000), phosphorylated-ERK1/2 (p-ERK1/2, $1: 1000)$, ERK1/2 (1:1000), and $\beta$-actin $(1: 2000)$. The membranes were incubated in horseradish peroxidase- (HRP-) conjugated secondary antibodies $(1: 2500)$. The blots were developed with the enhanced chemiluminescence (ECL) reagent.

2.10. Statistical Analysis. Data were presented as mean \pm standard error of the mean (SEM). The statistical significance was analyzed by one-way ANOVA with GraphPad Prism software. When ANOVA showed the significant differences, pairwise comparisons between means were accessed by Bonferroni's post hoc $t$-test with correction. A value of $p<0.05$ was considered statistically significant.

\section{Results}

3.1. TSG Produces Neuroprotection against 6-OHDA-Elicited DA Neurotoxicity In Vivo. After the intraperitoneal injection of TSG for 14 consecutive days, rat brains were collected, sectioned, and processed for quantification of DA neurons by immunohistochemistry staining with anti-TH antibody. As 

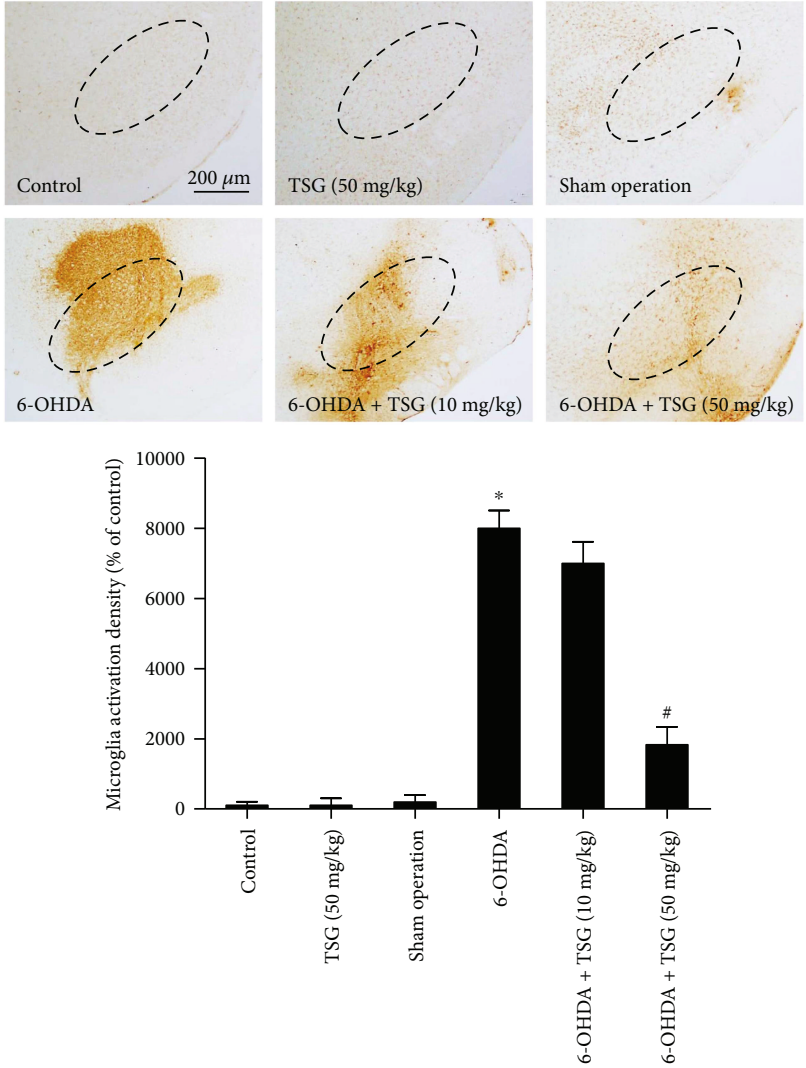

FIgURE 2: TSG attenuated 6-OHDA-induced microglial activation in vivo. Rat brain sections were immunostained by an anti-OX42 antibody. Densitometry analysis of nigral OX-42-positive microglia was performed with ImageJ software. The "ellipse" presented the area of SN. Scale bar $=200 \mu \mathrm{m}$. Quantified results were expressed as a percentage of the control group and were the mean \pm SEM from six rats. ${ }^{*} p<0.05$ compared with the control groups; ${ }^{\#} p<0.05$ compared with 6 -OHDA-treated group.

indicated in Figures 1(a) and 1(b), compared with the control group, 6-OHDA significantly reduced the TH-positive neurons in $\mathrm{SN}$, which was ameliorated by TSG treatment. In the rotarod test, TSG attenuated 6-OHDA-induced decrease in the time that the rats remained on the rod shown in Figure 1(c). In addition to DA neuronal number and rat behavior changes, the neuroprotective effects of TSG against 6-OHDA-induced depletion of striatum DA and DOPAC levels were further measured by HPLC coupled with electrochemical detection. As shown in Figures 1(d) and 1(e), in parallel with the reduction of DA neuronal number, a corresponding decrease in DA and DOPAC levels was discerned in 6-OHDA-treated group. However, TSG treatment attenuated 6-OHDA-caused decrease of DA and DOPAC levels in the striatum.

3.2. TSG Attenuates 6-OHDA-Induced Microglia Activation In Vivo. The brain sections were stained to detect the inhibitory effects of TSG on 6-OHDA-elicited microglia activation. As shown in Figure 2, 6-OHDA induced microglia activation compared with the control group. However, this activated state of microglia was attenuated by TSG treatment.
3.3. TSG Protects DA Neurons against 6-OHDA-Induced Neurotoxicity In Vitro. Primary rat midbrain neuron-glia cocultures were treated with TSG $(20-80 \mu \mathrm{M})$ for $30 \mathrm{~min}$ followed by the application of 6-OHDA $(40 \mu \mathrm{M})$. Seven days later, 6-OHDA-elicited DA damage was quantified by immunocytochemical staining and western blotting assay. As shown in Figure 3(a), TH-positive neuronal counting indicated that 6-OHDA significantly decreased DA neuronal number and TSG ameliorated 6-OHDA-elicited DA neuronal damage. Western blotting analysis also indicated that TSG attenuated 6-OHDA-elicited decrease of TH protein expression (Figure 3(b)). These in vitro results were in agreement with the findings from the in vivo studies.

3.4. TSG Ameliorates 6-OHDA-Elicited Microglia Activation In Vitro. Primary midbrain neuron-glia cocultures were pretreated with TSG $(20-80 \mu \mathrm{M})$ for $30 \mathrm{~min}$ and then stimulated by 6-OHDA. Seven days later, the 6-OHDAinduced microglia activation was analyzed. As shown in Figure 4(a), 6-OHDA significantly induced microglia activation. Furthermore, microglia in 6-OHDA-treated cultures presented an enlarged cell body and irregular shapes from the resting small and round cells to the highly activated amoeboid status. However, TSG significantly attenuated 6-OHDA-induced microglia activation. To further investigate the inhibitory properties of TSG on 6-OHDA-induced microglia activation, the whole cell lysis was collected and the effects of TSG on the protein expression of Iba1 (a marker of microglia used for western blot assay) were detected. As shown in Figure 4(b), 6-OHDA obviously increased Iba-1 protein expression, which was in parallel with TH-positive neuronal loss. TSG reduced 6-OHDAelevated Iba-1 protein expression.

3.5. TSG Decreases the Release of Proinflammatory Factors Induced by 6-OHDA. The inhibitory actions of TSG on the production of 6-OHDA-induced proinflammatory factors were further explored. Primary rat midbrain neuron-glia cocultures were treated with TSG $(20-80 \mu \mathrm{M})$ for $30 \mathrm{~min}$ followed by $6-\mathrm{OHDA}$ treatment. Twenty-four $\mathrm{h}$ later, the production of IL- $1 \beta$, NO, and TNF- $\alpha$ in the culture supernatant was detected by ELISA and Griess regent. As shown in Figure 5, the production of IL- $1 \beta$, NO, and TNF- $\alpha$ was increased in 6-OHDA-treated cultures. However, compared with 6-OHDA group, TSG significantly suppressed the production of the above proinflammatory factors.

3.6. TSG Attenuates 6-OHDA-Induced MAPK Pathway Activation. It is well known that MAPK signaling pathway participated in the modulation of immune responses. Next, the effects of TSG on 6-OHDA-induced MAPK pathway activation were evaluated. Primary neuron-glia cocultures were treated with TSG for $30 \mathrm{~min}$ followed by the stimulation of 6-OHDA for $24 \mathrm{~h}$. As shown in Figure 6, TSG attenuated 6-OHDA-induced ERK1/2 and p38 phosphorylation.

\section{Discussion}

This study demonstrated that TSG produced neuroprotection against 6-OHDA-induced DA neurotoxicity via the 

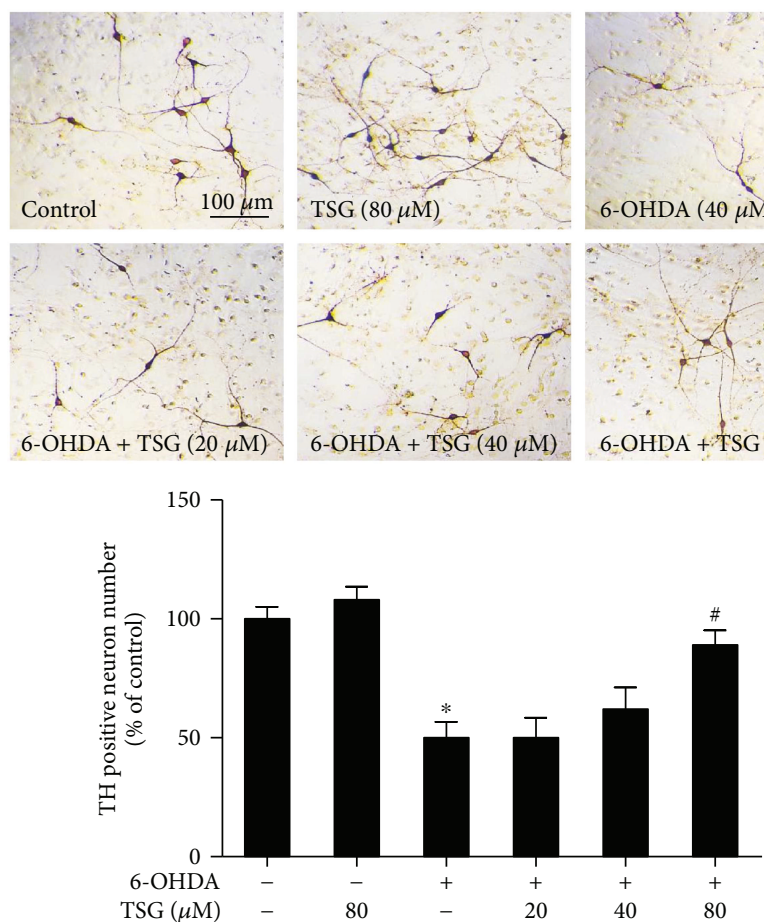

(a)
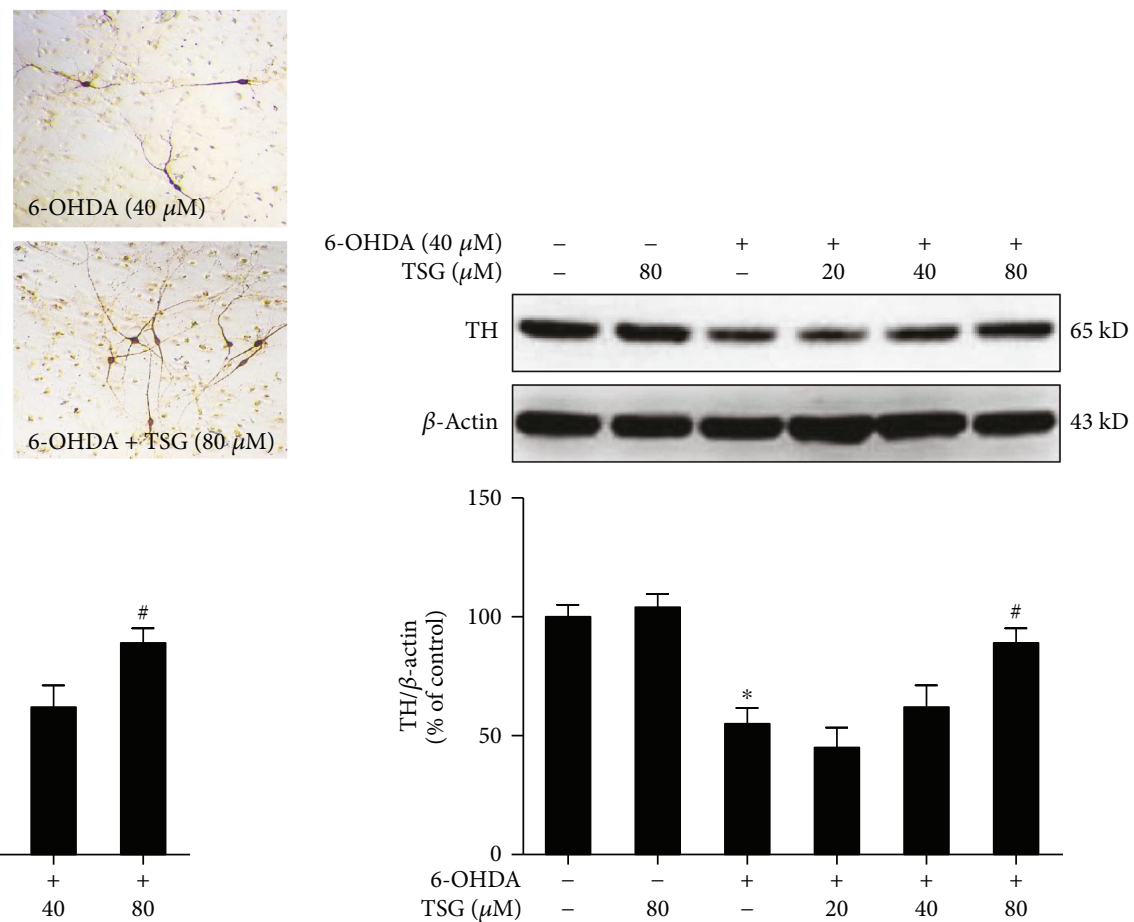

(b)

FIGURE 3: TSG protected DA neurons against 6-OHDA-induced neurotoxicity in vitro. 6-OHDA-induced DA neurotoxicity was quantified by $\mathrm{TH}$-positive neuron counting through the immunocytochemical analysis. Representative images of immunostaining from three experiments were shown (a). Scale bar $=100 \mu \mathrm{m}$. TH protein expression was determined by western blotting analysis. The ratio of densitometry values of $\mathrm{TH}$ with $\beta$-actin was assessed and normalized to each respective control group (b). Results were expressed as a percentage of the control group and were the mean \pm SEM from three independent experiments performed in triplicate. ${ }^{*} p<0.05$ compared with the control groups; ${ }^{\#} p<0.05$ compared with 6-OHDA-treated group.
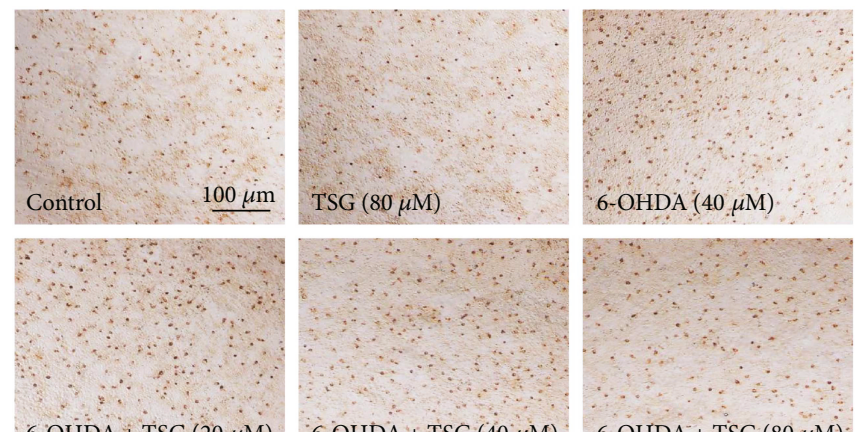

6-OHDA + TSG $(20 \mu \mathrm{M})$

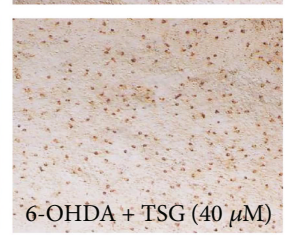

(a)
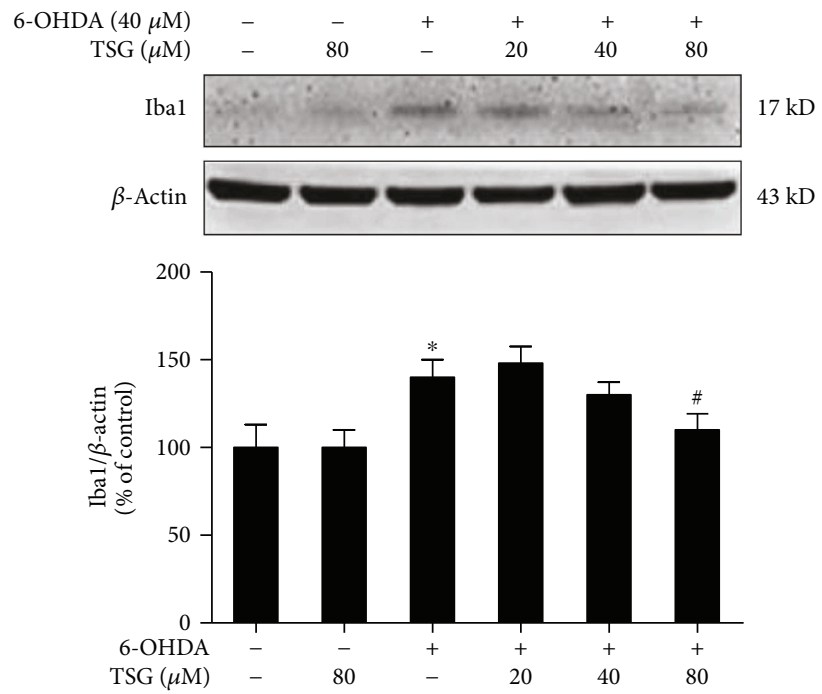

(b)

FIgURE 4: TSG ameliorated 6-OHDA-induced microglial activation in vitro. Activated microglia were detected by immunocytochemical staining with an anti-OX-42 antibody. Representative images of immunostaining from three experiments were indicated (a). Scale bar $=100 \mu \mathrm{m}$. Ibal protein expression was determined by western blotting assay. The ratio of densitometry values of Iba1 with $\beta$-actin was assessed and normalized to each respective control group (b). Results were expressed as a percentage of the control group and were the mean \pm SEM from three independent experiments performed in triplicate. ${ }^{*} p<0.05$ compared with the control groups; ${ }^{\#} p<0.05$ compared with 6-OHDA-treated group. 


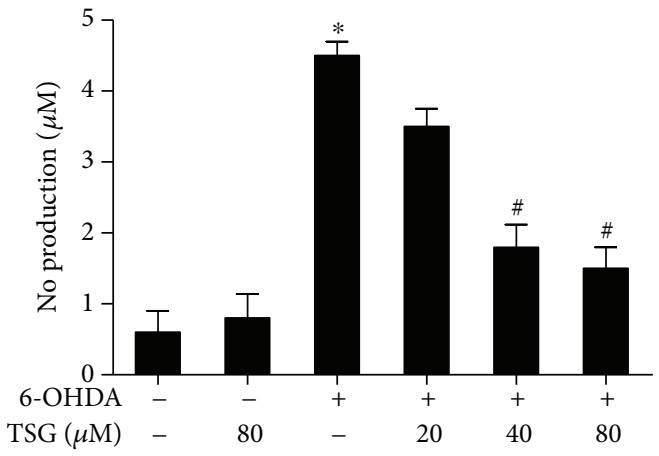

(a)

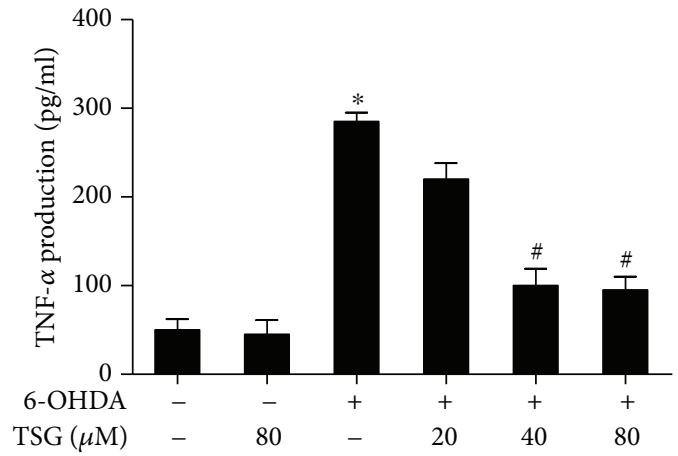

(b)

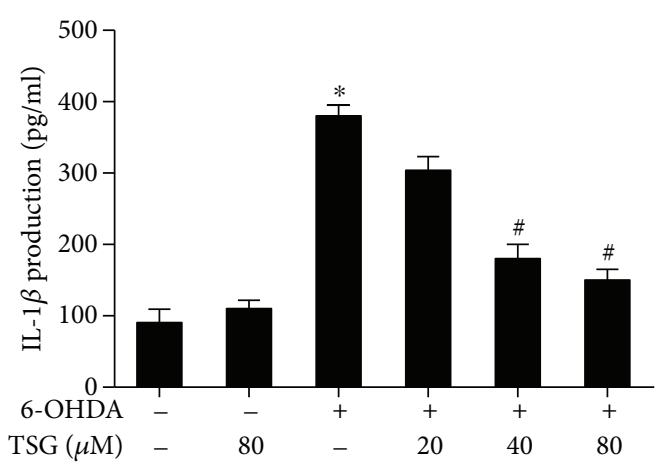

(c)

FIGURE 5: TSG suppressed the release of proinflammatory factors induced by 6-OHDA. The production of NO was determined by Griess reagent (a). The levels of TNF- $\alpha$ (b) and IL- $1 \beta$ (c) in the culture medium were detected by ELISA. Results were the mean \pm SEM from three independent experiments performed in triplicate. ${ }^{*} p<0.05$ compared with the control groups; ${ }^{*} p<0.05$ compared with 6 -OHDAtreated group.

attenuation of microglia activation and the subsequent release of proinflammatory factors. These findings suggest that TSG might hold potential therapeutic effects on PD.

Recently, neuroinflammation has been confirmed to be involved in the etiology and pathogenesis of PD [14]. Microglia activation that participated in the modulation of neuroinflammation has been well documented [15]. Upon activation by brain injury and inflammogen stimuli, microglia excreted amounts of proinflammatory factors, such as cytokines and reactive oxygen species (ROS). The accumulation of these factors was recognized to lead to the progressive loss of DA neurons [16]. However, the dying/dead DA neurons also release various types of neurotoxic soluble factors such as $\alpha$-synuclein and damage-associated molecular patterns (DAMPs), which in turn induced microglia reactivation. These activated microglia again released proinflammatory factors and caused the continuous DA neuronal damage [17]. Thus, a "self-propelling" vicious cycle was created to result in the gradually progressive DA neurodegeneration. 6-OHDA, a hydroxyl derivative of DA, competes for dopamine uptake sites and oxidized to neurotoxic substances upon injection into the SN of the brain. Neurotoxic action of 6-OHDA occurs through the accumulation of the toxin in DA neurons, followed by the alteration of cellular homeostasis and neuronal damage [18]. Till now, 6-OHDA-elicited DA neurodegeneration was a well-characterized experimental PD model both in vivo and in vitro [19]. In the present study, 6-OHDA induced DA neurons loss and then indirectly elicited microglia activation and consequent proinflammatory factor excretion. However, TSG seemed to produce DA neuroprotection via the inhibition of microglia activation and thus halt this vicious cycle running.

Next, to explore whether TSG was capable of reducing microglia-mediated neuroinflammatory response, the effects of TSG on NO, IL- $1 \beta$, and TNF- $\alpha$ production were determined. Increasing evidence has demonstrated that the proinflammatory factors such as NO, IL-1 $\beta$, and TNF $\alpha$ contributed to neurodegenerative diseases [20]. Postmortem data indicated that the extensive proliferation of reactive microglia and the elevated production of NO, IL- $1 \beta$, and $\mathrm{TNF}-\alpha$ were discerned in the SN region [21]. In addition, NO could react with ROS to form highly toxic intermediates such as peroxynitrite to induce DA neuronal damage [22]. Thus, inhibition of neuroinflammatory factor production might exhibit a promising therapeutic potential for PD. In line with our previous findings that TSG inhibited LPSelicited proinflammatory factor production in BV2 cells [23], the present study demonstrated that TSG reduced 6OHDA-elicited proinflammatory factor release. These findings suggested that amelioration of microglia activation and reduction of the subsequent proinflammatory factor generation were involved in TSG-mediated neuroprotection against 6-OHDA-induced DA neurotoxicity.

MAPK pathway is a well-known vital regulator of neuroinflammation [24]. Intensive studies showed that 6-OHDAinduced DA neuronal injury has been attributed to the 


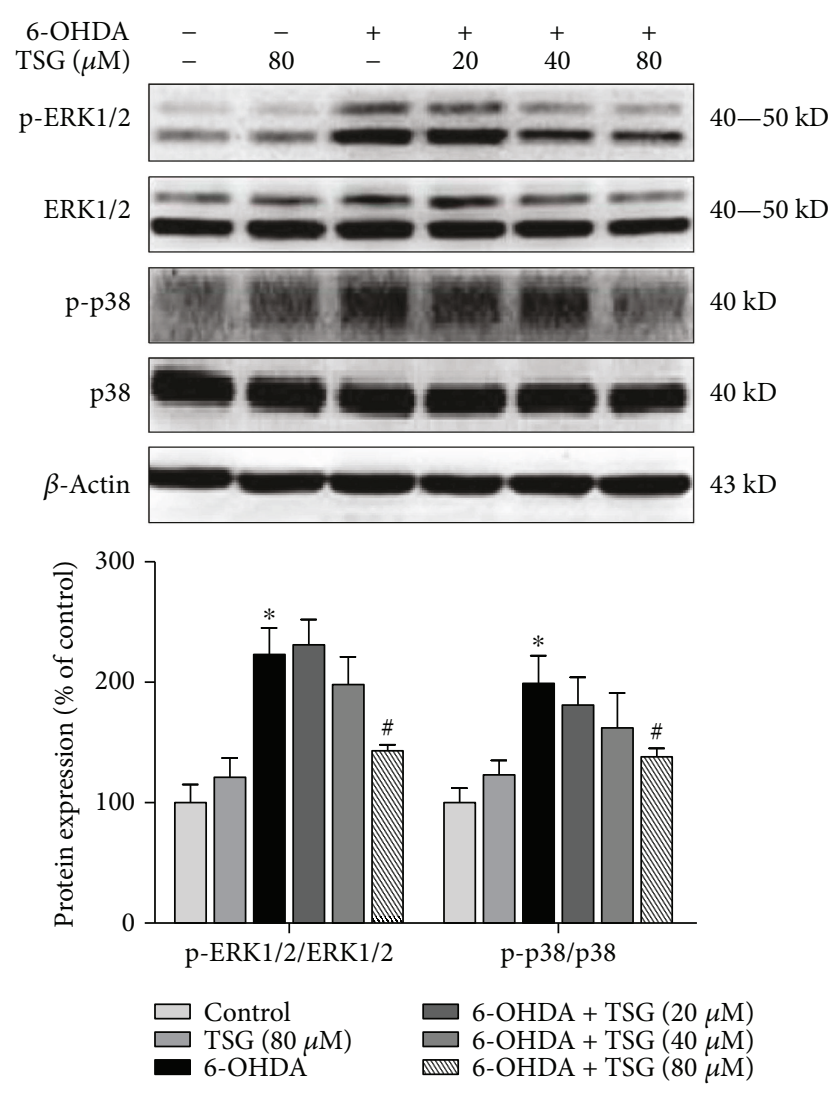

FIGURE 6: TSG attenuated 6-OHDA-induced MAPK pathway activation. The protein expressions of $\mathrm{p}$-ERK1/2, ERK1/2, pp38, and p38 were investigated by western blotting analysis. The ratio of densitometry values of p-ERK1/2 and p-p38 with total ERK1/2 and p38 was analyzed and normalized to each respective control group. Results were expressed as mean \pm SEM from three independent experiments performed in triplicate. ${ }^{*} p<0.05$ compared with the control groups; ${ }^{\#} p<0.05$ compared with 6OHDA-treated group.

activation of MAPK pathway [25]. Moreover, MAPK pathway regulates the release of proinflammatory cytokines from activated microglia including NO, IL- $1 \beta$, and TNF- $\alpha$ [26]. Two members of the MAPK family, p38 and ERK1/2, were all activated upon neuroinflammation. Amounts of studies confirmed that ERK1/2 and p38 had been indicated to mediate neuroinflammatory reactions in $\mathrm{AD}$ and $\mathrm{PD}$ models $[27,28]$. ERK1/2 can be activated by oxidative stress, inflammation, and cytokines [29], and a specific ERK1/2 inhibitor, U0126, conferred significant neuroprotection against DA neurodegeneration [30]. Besides, a series of studies have illustrated that the damaged DA neurons were accompanied by the increased number of p-p38 and iNOS-positive cells in the SN of PD mice model [31]. Therefore, the intervention of MAPK pathway activation is consequently applied for neurodegenerative disease treatment [32]. This study found that TSG inhibited 6-OHDA-elicited activation of MAPK pathway, implying that the inhibitory effects of TSG on proinflammatory factor production would likely result from the inactivation of MAPK pathway.

At present, current available drugs alleviate the symptoms of PD but are inadequate for halting the progression of PD. However, the adverse effects of the available drugs and nonmotor symptoms hold huge challenges for longterm therapy. Therefore, the new potential therapeutic agents and strategies are urgently required to delay or stop the progressive features of the disease [33]. Since neuroinflammation is considered to contribute to the acceleration of $\mathrm{PD}$ progression, this process might be an important breakpoint for the disease control. Thus, treatment with anti-inflammatory agents is gaining importance on the pharmacotherapy of PD. The present study revealed that TSG produced DA neuroprotection via its anti-inflammatory actions. However, further studies are required to fully illuminate the molecular mechanisms through which TSG potentiates anti-inflammatory activities.

\section{Conclusion}

This study here demonstrates that TSG produces neuroprotection from 6-OHDA-induced DA neurotoxicity through the inhibition of microglia-elicited neuroinflammation. Our data indicate that TSG might possess a beneficial potential for PD treatment.

\section{Conflicts of Interest}

The authors declare no conflicts of interests.

\section{Authors' Contributions}

Chun Huang and Fengqin Lin contributed equally to this work.

\section{Acknowledgments}

This study was supported by the National Natural Science Foundation of China (nos. 81102433 and 81460556), the Foundation for High-Level Innovative Talents of Guizhou (no. 20164027), the Governor Talent Foundation of Guizhou (no. 201288), the Innovation Research Group Project of Education Department of Guizhou (no. 2016038), and the Foundation for Excellent Young Talents of Zunyi Medical University.

\section{References}

[1] C. M. Lema Tomé, T. Tyson, N. L. Rey, S. Grathwohl, M. Britschgi, and P. Brundin, "Inflammation and $\alpha$ synuclein's prion-like behavior in Parkinson's disease-is there a link?," Molecular Neurobiology, vol. 47, no. 2, pp. 561-574, 2013.

[2] M. Nilashi, O. Ibrahim, and A. Ahani, "Accuracy improvement for predicting Parkinson's disease progression," Scientific Reports, vol. 6, no. 1, article 34181, 2016.

[3] E. C. Hirsch, S. Vyas, and S. Hunot, "Neuroinflammation in Parkinson's disease," Parkinsonism \& Related Disorders, vol. 18, Supplement 1, pp. S210-S212, 2012.

[4] H. M. Gao and J. S. Hong, "Why neurodegenerative diseases are progressive: uncontrolled inflammation drives disease progression," Trends in Immunology, vol. 29, no. 8, pp. 357365, 2008. 
[5] X. F. Wu, M. L. Block, W. Zhang et al., "The role of microglia in paraquat-induced dopaminergic neurotoxicity," Antioxidants \& Redox Signaling, vol. 7, no. 5-6, pp. 654-661, 2005.

[6] W. Zhang, C. H. Wang, F. Li, and W. Z. Zhu, " $2,3,4^{\prime}, 5-$ Tetrahydroxystilbene- $2-O-\beta$-D-glucoside suppresses matrix metalloproteinase expression and inflammation in atherosclerotic rats," Clinical and Experimental Pharmacology and Physiology, vol. 35, no. 3, pp. 310-316, 2008.

[7] T. Wang, J. Gu, P. F. Wu et al., "Protection by tetrahydroxystilbene glucoside against cerebral ischemia: involvement of JNK, SIRT1, and NF- $\kappa$ B pathways and inhibition of intracellular ROS/RNS generation," Free Radical Biology \& Medicine, vol. 47, no. 3, pp. 229-240, 2009.

[8] C. Shen, F.-1. Sun, R.-y. Zhang et al., “Tetrahydroxystilbene glucoside ameliorates memory and movement functions, protects synapses and inhibits $\alpha$-synuclein aggregation in hippocampus and striatum in aged mice," Restorative Neurology and Neuroscience, vol. 33, no. 4, pp. 531-541, 2015.

[9] L. Zhang, L. H. Huang, L. Chen, D. Hao, and J. Chen, "Neuroprotection by tetrahydroxystilbene glucoside in the MPTP mouse model of Parkinson's disease," Toxicology Letters, vol. 222, no. 2, pp. 155-163, 2013.

[10] J. Hou, Q. Liu, Y. Li, H. Sun, and J. Zhang, "An in vivo microdialysis study of FLZ penetration through the bloodbrain barrier in normal and 6-hydroxydopamine induced Parkinson's disease model rats," BioMed Research International, vol. 2014, Article ID 850493, 11 pages, 2014.

[11] G. Khuwaja, M. M. Khan, T. Ishrat et al., "Neuroprotective effects of curcumin on 6-hydroxydopamine-induced parkinsonism in rats: behavioral, neurochemical and immunohistochemical studies," Brain Research, vol. 1368, pp. 254-263, 2011.

[12] W. Zhang, E. J. Shin, T. Wang et al., "3-Hydroxymorphinan, a metabolite of dextromethorphan, protects nigrostriatal pathway against MPTP-elicited damage both in vivo and in vitro," The FASEB Journal, vol. 20, no. 14, pp. 24962511, 2006.

[13] F. Zhang, L. Qian, P. M. Flood, J. S. Shi, J. S. Hong, and H. M. Gao, "Inhibition of $\mathrm{I} \kappa \mathrm{B}$ kinase- $\beta$ protects dopamine neurons against lipopolysaccharide-induced neurotoxicity," The Journal of Pharmacology and Experimental Therapeutics, vol. 333, no. 3, pp. 822-833, 2010.

[14] A. De Virgilio, A. Greco, G. Fabbrini et al., "Parkinson's disease: autoimmunity and neuroinflammation," Autoimmunity Reviews, vol. 15, no. 10, pp. 1005-1011, 2016.

[15] V. Sanchez-Guajardo, C. J. Barnum, M. G. Tansey, and M. Romero-Ramos, "Neuroimmunological processes in Parkinson's disease and their relation to $\alpha$-synuclein: microglia as the referee between neuronal processes and peripheral immunity," ASN Neuro, vol. 5, no. 2, pp. AN20120066AN20120139, 2013.

[16] M. L. Block, L. Zecca, and J. S. Hong, "Microglia-mediated neurotoxicity: uncovering the molecular mechanisms," Nature Reviews Neuroscience, vol. 8, no. 1, pp. 57-69, 2007.

[17] S. H. Chen, E. A. Oyarzabal, and J. S. Hong, "Critical role of the Mac1/NOX2 pathway in mediating reactive microgliosis-generated chronic neuroinflammation and progressive neurodegeneration," Current Opinion in Pharmacology, vol. 26, pp. 54-60, 2016.

[18] H.-J. Im, J. Hahm, H. Kang et al., "Disrupted brain metabolic connectivity in a 6-OHDA-induced mouse model of Parkinson's disease examined using persistent homology- based analysis," Scientific Reports, vol. 6, no. 1, article 33875, 2016.

[19] A. Wasik, D. Polak, I. Romanska, J. Michaluk, and L. Antkiewicz-Michaluk, "The impact of 1MeTIQ on the dopaminergic system function in the 6-OHDA model of Parkinson's disease," Pharmacological Reports, vol. 68, no. 6, pp. 1205-1213, 2016.

[20] M. C. Pott Godoy, R. Tarelli, C. C. Ferrari, M. I. Sarchi, and F. J. Pitossi, "Central and systemic IL-1 exacerbates neurodegeneration and motor symptoms in a model of Parkinson's disease," Brain, vol. 131, no. 7, pp. 1880-1894, 2008.

[21] Y. Choi, M. K. Lee, S. Y. Lim, S. H. Sung, and Y. C. Kim, "Inhibition of inducible NO synthase, cyclooxygenase-2 and interleukin- $1 \beta$ by torilin is mediated by mitogen-activated protein kinases in microglial BV2 cells," British Journal of Pharmacology, vol. 156, no. 6, pp. 933-940, 2009.

[22] H. M. Gao, F. Zhang, H. Zhou, W. Kam, B. Wilson, and J. S. Hong, "Neuroinflammation and $\alpha$-synuclein dysfunction potentiate each other, driving chronic progression of neurodegeneration in a mouse model of Parkinson's disease," Environmental Health Perspectives, vol. 119, no. 6, pp. 807-814, 2011.

[23] F. Zhang, Y.-Y. Wang, J. Yang, Y.-F. Lu, J. Liu, and J.-S. Shi, "Tetrahydroxystilbene glucoside attenuates neuroinflammation through the inhibition of microglia activation," Oxidative Medicine and Cellular Longevity, vol. 2013, Article ID 680545, 8 pages, 2013.

[24] R. Herrera-Molina, B. Flores, J. A. Orellana, and R. von Bernhardi, "Modulation of interferon- $\gamma$-induced glial cell activation by transforming growth factor $\beta 1$ : a role for STAT1 and MAPK pathways," Journal of Neurochemistry, vol. 123, no. 1, pp. 113-123, 2012.

[25] C. J. Hwang, H. P. Lee, D. Y. Choi et al., "Inhibitory effect of thiacremonone on MPTP-induced dopaminergic neurodegeneration through inhibition of p38 activation," Oncotarget, vol. 7, no. 30, pp. 46943-46958, 2016.

[26] H. Liu, Y. Deng, J. Gao et al., "Sodium hydrosulfide attenuates beta-amyloid-induced cognitive deficits and neuroinflammation via modulation of MAPK/NF- $\kappa$ B pathway in rats," Current Alzheimer Research, vol. 12, no. 7, pp. 673-683, 2015.

[27] H. S. Um, E. B. Kang, J. H. Koo et al., "Treadmill exercise represses neuronal cell death in an aged transgenic mouse model of Alzheimer's disease," Neuroscience Research, vol. 69, no. 2, pp. 161-173, 2011.

[28] I. G. Onyango, J. B. Tuttle, and J. P. Bennett Jr., "Activation of p38 and $\mathrm{N}$-acetylcysteine-sensitive c-Jun NH2-terminal kinase signaling cascades is required for induction of apoptosis in Parkinson's disease cybrids," Molecular and Cellular Neurosciences, vol. 28, no. 3, pp. 452-461, 2005.

[29] L. Qian, S.-J. Wei, D. Zhang et al., "Potent anti-inflammatory and neuroprotective effects of TGF- $\beta 1$ are mediated through the inhibition of ERK and $\mathrm{p} 47^{\text {phox }}-\mathrm{Ser}^{345}$ phosphorylation and translocation in microglia," The Journal of Immunology, vol. 181, no. 1, pp. 660-668, 2008.

[30] R. L. Miller, M. James-Kracke, G. Y. Sun, and A. Y. Sun, "Oxidative and inflammatory pathways in Parkinson's disease," Neurochemical Research, vol. 34, no. 1, pp. 55-65, 2009.

[31] Q. Wang, H. Zhang, M. Liu et al., "P38 MAPK signaling pathway regulates nuclear factor- $\kappa \mathrm{B}$ and inducible nitric oxide synthase expressions in the substantia nigra in a mouse model of Parkinson's disease," Nan Fang Yi Ke Da Xue Xue Bao, vol. 34 , no. 8, pp. 1176-1180, 2014. 
[32] Y. H. Jeong, Y.-C. Oh, W.-K. Cho, N.-H. Yim, and J. Y. Ma, "Anti-inflammatory effect of rhapontici radix ethanol extract via inhibition of NF- $\kappa$ B and MAPK and induction of HO1 in macrophages," Mediators of Inflammation, vol. 2016, Article ID 7216912, 13 pages, 2016.

[33] K. Strecker and J. Schwarz, "Parkinson's disease: emerging pharmacotherapy," Expert Opinion on Emerging Drugs, vol. 13, no. 4, pp. 573-591, 2008. 


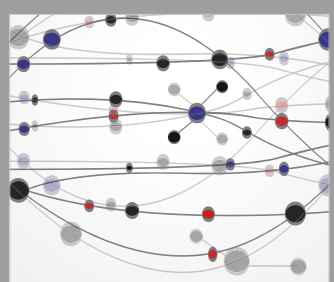

The Scientific World Journal
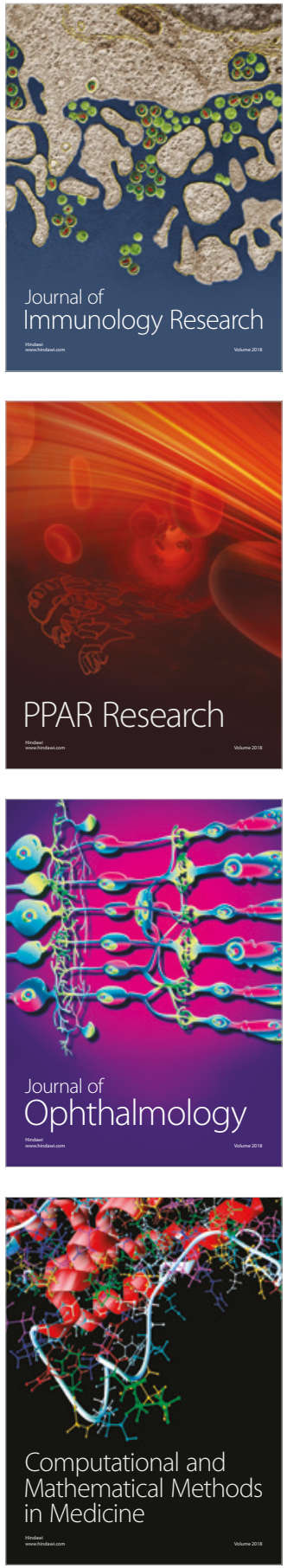

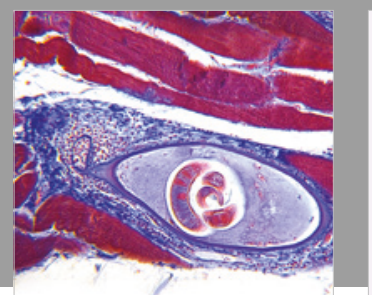

Gastroenterology Research and Practice

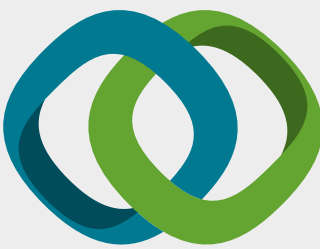

\section{Hindawi}

Submit your manuscripts at

www.hindawi.com
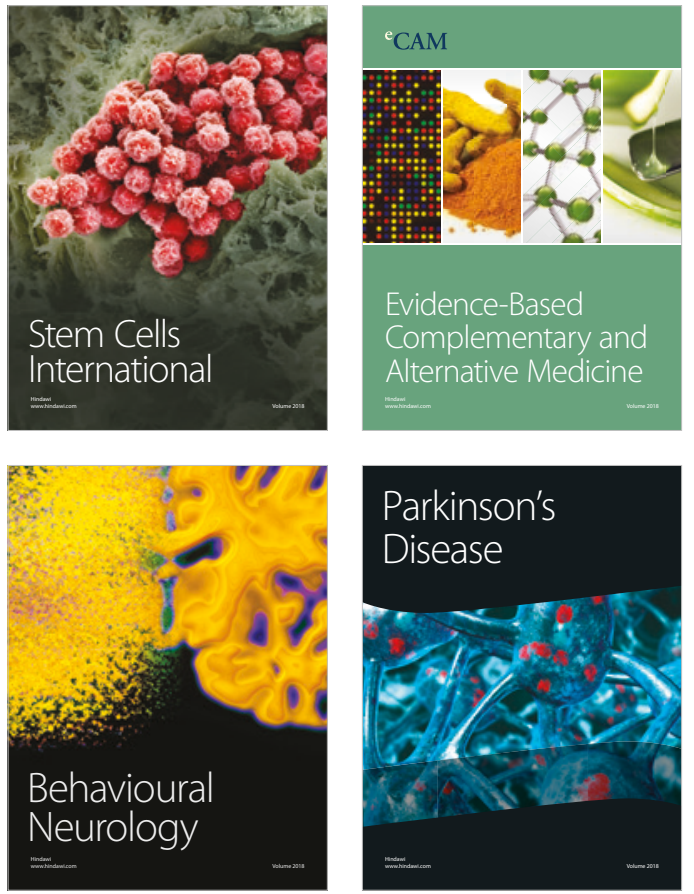

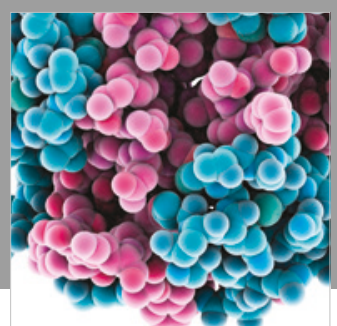

ournal of

Diabetes Research

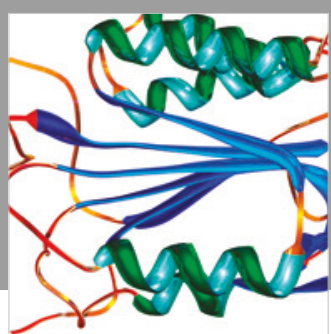

Disease Markers
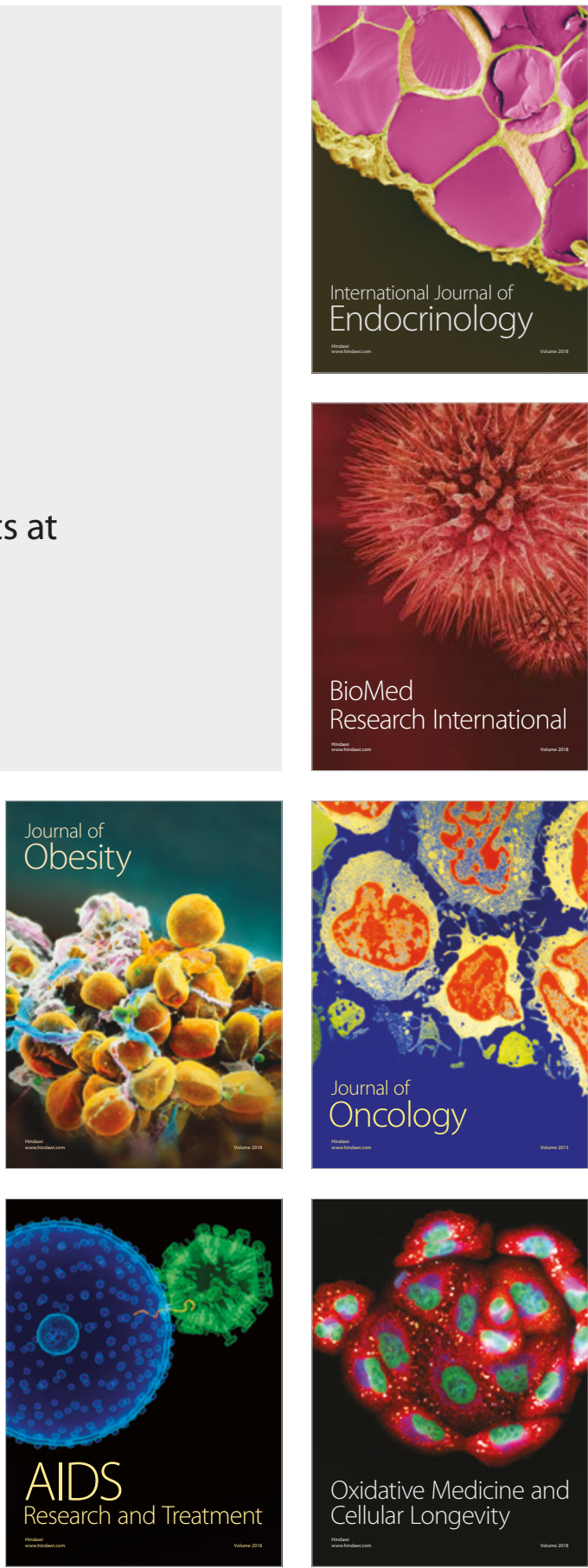